\title{
Endothelial Connexin37 and Connexin40 participate in basal but not agonist-induced NO release
}

Merlijn J Meens ${ }^{1,2^{*}}$, Florian Alonso ${ }^{3}$, Loïc Le Gal ${ }^{3}$, Brenda R Kwak ${ }^{1,2+}$ and Jacques-Antoine Haefliger ${ }^{3+}$

\begin{abstract}
Background: Connexin37 (Cx37) and Cx40 are crucial for endothelial cell-cell communication and homeostasis. Both connexins interact with endothelial nitric oxide synthase (eNOS). The exact contribution of these interactions to the regulation of vascular tone is unknown.

Results: Cx37 and Cx40 were expressed in close proximity to eNOS at cell-cell interfaces of mouse aortic endothelial cells. Absence of Cx37 did not affect expression of Cx40 and a $50 \%$ reduction of Cx40 in Cx40 ${ }^{+/-}$aortas did not affect the expression of $\mathrm{Cx} 37$. However, absence of $\mathrm{C} \times 40$ was associated with reduced expression of $\mathrm{Cx37}$. Basal $\mathrm{NO}$ release and the sensitivity for ACh were decreased in $\mathrm{C}_{3} 7^{-/-}$and $\mathrm{C} \times 40^{-/-}$aortas but not in $\mathrm{C} \times 40^{+/-}$aortas. Moreover, ACh-induced release of constricting cyclooxygenase products was present in WT, $\mathrm{C} \times 40^{-/-}$and $\mathrm{C} \times 40^{+/-}$aortas but not in $\mathrm{C} \times 37^{-/-}$aortas. Finally, agonist-induced NO-dependent relaxations and the sensitivity for exogenous NO were not affected by genotype.
\end{abstract}

Conclusions: Cx37 is more markedly involved in basal NO release, release of cyclooxygenase products and the regulation of the sensitivity for ACh as compared to $\mathrm{C} \times 40$.

Keywords: Connexin, Nitric oxide, Endothelium

\section{Background}

Connexins form a family of transmembrane proteins $[1,2]$. They are inserted into cell membranes as hexamers called connexons. Gap junction channels, structures that arise after docking of two connexons from neighbouring cells, allow diffusion of ions and small metabolites (e.g. ATP, $\mathrm{Ca}^{2+}$ and cAMP) between cells and are thus implicated in direct cell-to-cell communication and synchronization of tissue responses [3]. Under specific conditions, connexons may also function as hemichannels allowing for diffusion of ions and small metabolites from the cytosol to the extracellular space or vice versa [4].

\footnotetext{
* Correspondence: merlijn.meens@unige.ch

${ }^{\dagger}$ Equal contributors

'Department of Pathology and Immunology, University of Geneva, 6th floor,

1 Rue Michel-Servet, 1211 Geneva, Switzerland

${ }^{2}$ Department of Medical Specialties - Cardiology, University of Geneva,

Geneva, Switzerland

Full list of author information is available at the end of the article
}

It is now increasingly recognized that connexins do not only function as connexons or gap junction channels; they can also regulate the function of other proteins via protein-protein interactions [5-7]. It has for instance been demonstrated that $\mathrm{Cx} 37$ interacts with eNOS [8]. Moreover, these interactions between Cx37 and eNOS reduce basal nitric oxide $(\mathrm{NO})$ release in vitro [8]. However, a specific role for Cx37-eNOS interactions at the organ level remains to be addressed. In addition, it has been shown that $\mathrm{Cx} 40$ interacts with eNOS and that Cx40-deficient mice are characterized by reduced endothelium-dependent NO-mediated relaxations [9]. However, Cx37 is also reduced in Cx40-deficient mice [9]. It is thus unclear whether Cx37-eNOS interactions, Cx40-eNOS interactions or both are responsible for the vascular phenotype of Cx40-deficient mice. Therefore, the current study addressed whether Cx37-deficiency, Cx40-deficiency or a $50 \%$ reduced expression of Cx40 affects basal or agonist-induced release of NO. The key findings are that mainly $\mathrm{Cx} 37$ directly modulates i) the spontaneous release of $\mathrm{NO}$ from mouse aortic 
endothelium, ii) the sensitivity of mouse aortic endothelial cells for ACh and iii) the agonist-induced release of endothelium-derived COX-generated contractile factors.

\section{Results and discussion}

Mouse aortic endothelial cells express $\mathrm{C} \times 37$ and $\mathrm{C} \times 40$ but not Cx43s

The cellular localization of $\mathrm{Cx} 37, \mathrm{Cx} 40$ and $\mathrm{Cx} 43$ was studied by immunofluorescence performed on en face preparations of WT, $\mathrm{Cx} 37^{-/-}$and $\mathrm{C} \times 40^{-/-}$aortas. These experiments showed that WT mouse aortic endothelial cells expressed $\mathrm{Cx} 37$ and $\mathrm{Cx} 40$ at cell-cell interfaces whereas Cx43 was barely detectable (Fig. 1a-c). In $\mathrm{C} \times 37^{-1-}$ endothelium, Cx37 and Cx43 were not detected and the immunosignal for $\mathrm{Cx} 40$ was comparable to the Cx40 immunosignal in WT endothelium (Fig. 1d-f). Finally, in $\mathrm{Cx} 40^{-1-}$ endothelium, Cx40 and Cx43 were not found and the immunosignal for Cx37 was reduced as compared to the Cx37 immunosignal in WT endothelium (Fig. 1g-i). In summary, i) Cx37 and $\mathrm{Cx} 40$ are expressed at intercellular junctions of mouse aortic endothelial cells, ii) the level of $\mathrm{Cx} 37$ expression seems to be dependent on the expression of Cx40 and iii) Cx43 is barely detectable in mouse aortic endothelium. Expression of $\mathrm{Cx} 43$ in rat aortic endothelium is mainly restricted to areas exposed to disturbed blood flow [10], hence a low level of Cx43 expression in mouse aortic endothelial cells of the thoracic aorta was in line with expectations. Moreover, this study was performed on a part of the thoracic aorta that is exposed to high laminar shear stress, a condition that likely increases expression of Cx37 expression due to its effect on the transcription factor KLF2 [11] and that might increase expression of $\mathrm{Cx} 40$ due to the activation of Akt similar to the situation in arterioles [12]. Interestingly, the immunosignal for $\mathrm{Cx} 37$ seemed reduced in $\mathrm{Cx} 40^{--}$mouse aortic endothelial cells whereas the immunosignal for $\mathrm{Cx} 40$ was not altered in $\mathrm{Cx}_{3} 7^{-/-}$endothelium. Thus, there might be interdependence of Cx37 and Cx40 expression.

\section{Cx40-deficiency affects endothelial Cx37 expression}

To further study whether expression of Cx37 affects the expression of Cx40 or vice versa, the expression of Gja4 (the gene coding for mouse Cx37) or Gja5 (the gene coding for mouse $\mathrm{Cx} 40$ ) was quantified by real-time PCR performed on total aortic mRNA. As expected, mRNA coding for Cx37 was detectable in WT aortas but not in $\mathrm{Cx} 37^{-/-}$aortas (Fig. 2a). Moreover, mRNA coding

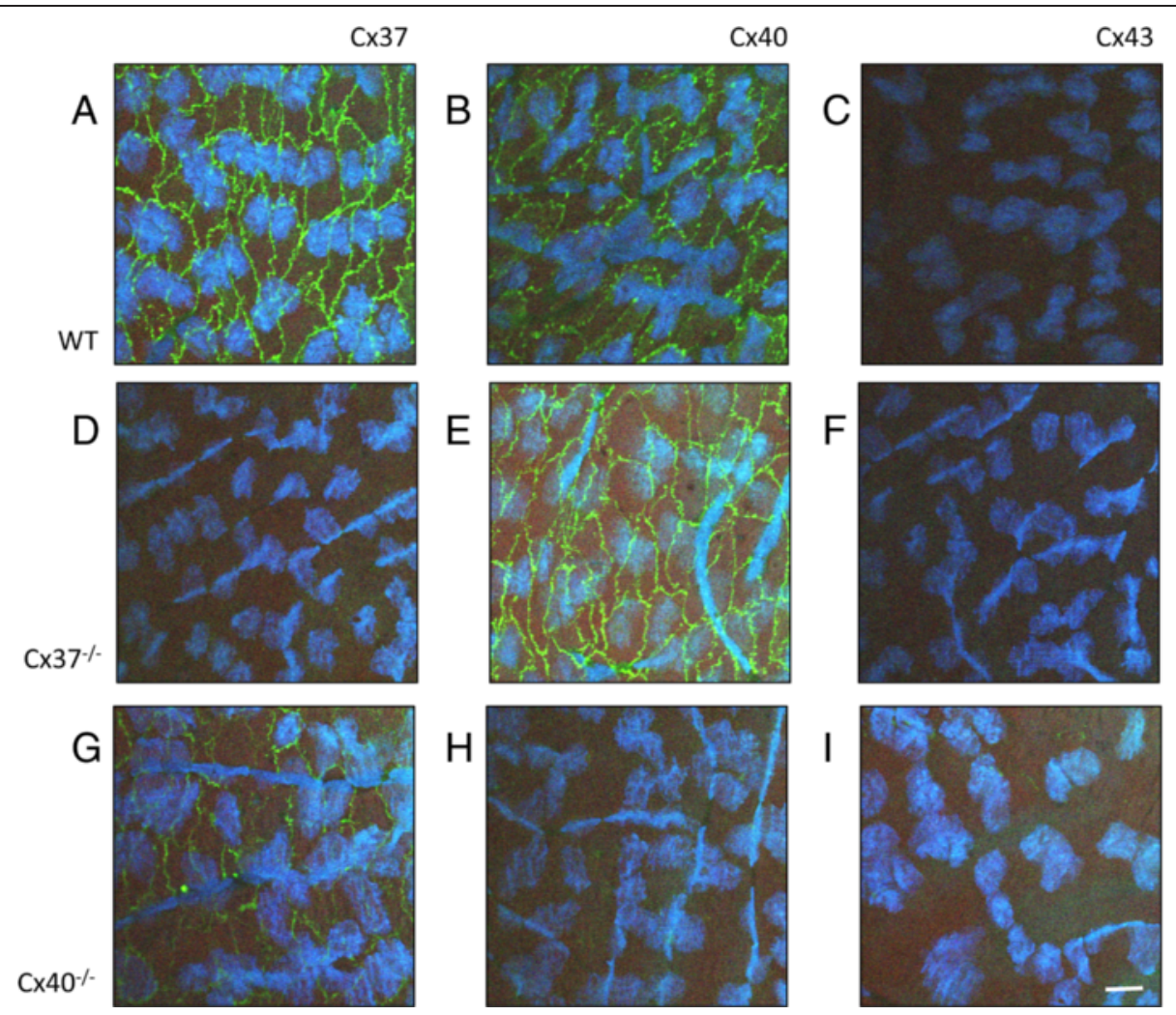

Fig. 1 Mouse aortic endothelial cells express Cx37 and Cx40 at cell-cell interfaces. a-c Representative images of en face confocal immunofluorescent stainings for Cx37 (a), Cx40 (b) or Cx43 (c) in wild-type (WT) mouse aortic endothelium, respectively. d-f Representative images of en face confocal immunofluorescent stainings for Cx37 (d), Cx40 (e) or Cx43 (f) in Cx37-/- mouse aortic endothelium, respectively. $\mathbf{g}$-i Representative images of en face confocal immunofluorescent stainings for $\mathrm{C} \times 37$ (g), $\mathrm{C} \times 40$ (h) or $\mathrm{C} \times 43$ (i) in $\mathrm{C} \times 40^{-/-}$mouse aortic endothelium, respectively. Scalebar equals $15 \mu \mathrm{M}$ 

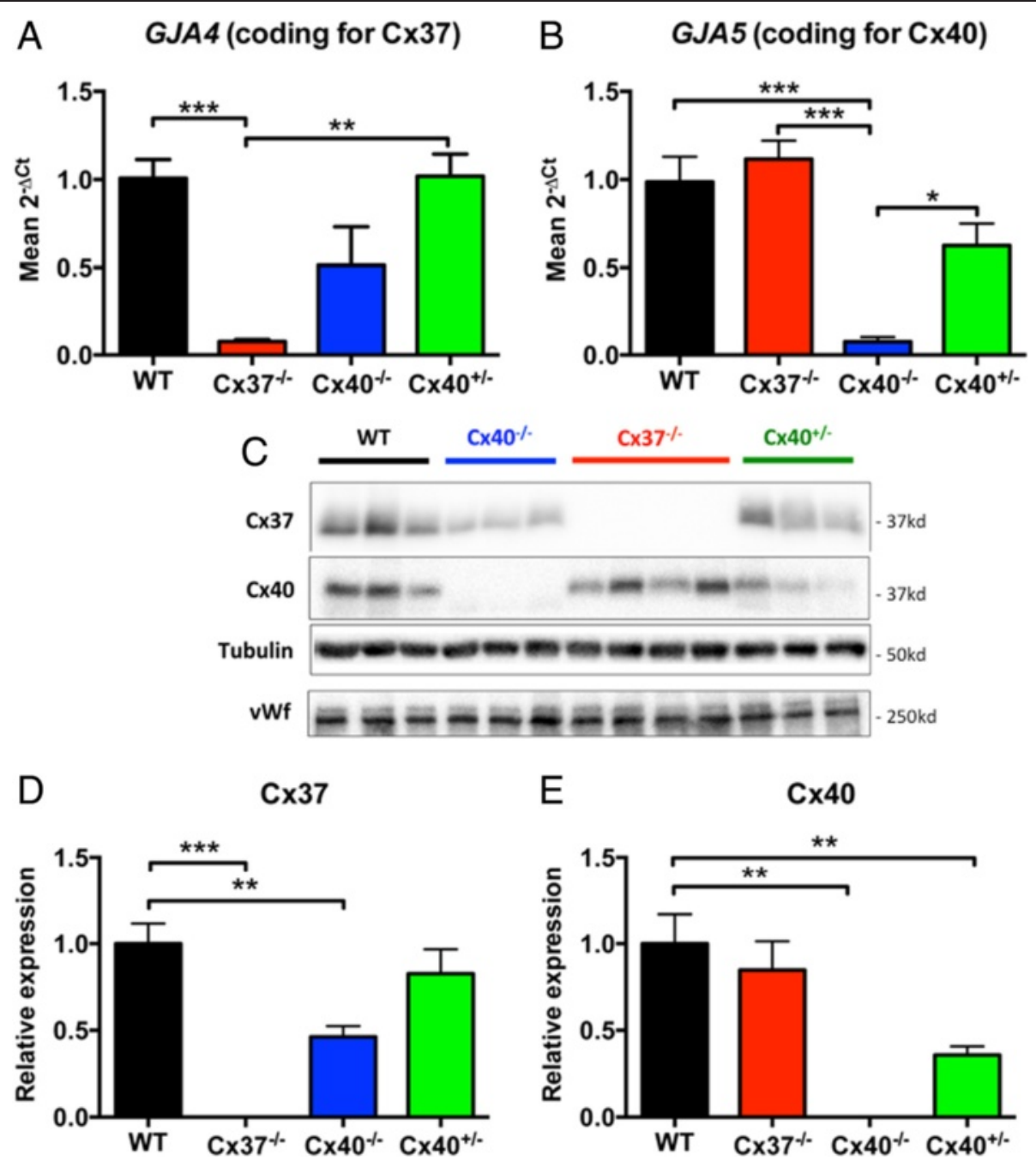

Fig. 2 Cx37-deficiency does not affect expression of $C \times 40$ while $C \times 40$-deficiency reduces expression of $\mathbf{C x 3 7}$. a-b Total mRNA was isolated from aortas obtained from WT, $\mathrm{C} \times 37^{-1-}, \mathrm{C} \times 40^{-1-}$ or $\mathrm{C} \times 40^{+/-}$mice $(N=3)$. Thereafter, the expression of Gja4 (A, the gene coding for $\left.\mathrm{C} \times 37\right)$ or $\mathrm{Gja}$ 5 (B, the gene coding for $\mathrm{C} \times 40$ ) was assessed by real-time PCR. Cx40 is expressed at WT levels in the $\mathrm{C} \times 37^{-1-}$ aortas while $\mathrm{C} \times 37$ showed large variation and tended to be reduced in the $\mathrm{C} \times 40^{-/-}$aortas $(p=0.08)$. Moreover, expression of $\mathrm{C} \times 37$ is not reduced in $\mathrm{C} \times 40^{+/-}$aortas. c Representative image of $\mathrm{C} \times 37$ and $\mathrm{C} \times 40$ protein expression in scraped mouse aortic endothelial cells as assessed by western blot. Tubulin and von Willebrand factor (vWf) were assessed for equal protein loading. $\mathbf{d}$ Cx37 protein quantification $(N=6-8)$. Similar to the expression of Cx37 at the level of RNA, Cx37 expression was reduced by half in $\mathrm{C} \times 40^{-1-}$ aortic endothelial cells whereas $\mathrm{C} \times 37$ expression was not significantly reduced in $\mathrm{C} \times 40^{+/-}$endothelium. e $\mathrm{C} \times 40$ protein quantification ( $N=6-8)$. $C \times 40$ was diminished by half in $\mathrm{C} \times 40^{+/-}$endothelial cells and was not altered in $\mathrm{C} \times 37^{-/-}$endothelium. Values are expressed as mean \pm SEM. ${ }^{*},{ }^{* *}$ or ${ }^{* * *} p<0.05,0.01$ or 0.001 vs WT control, respectively

for Cx37 showed a large variation and tended to be reduced in $\mathrm{Cx} 40^{-/-}$aortas $(p=0.08)$ and was comparable to WT levels in $\mathrm{Cx} 40^{+/-}$aortas (Fig. 2a). These findings are in line with an earlier study showing that endothelial Cx40-deficient mice do not display significantly reduced Cx37 mRNA levels in both the aorta and the cremaster muscle [13]. mRNA coding for Cx40 was expressed at similar levels in WT and $\mathrm{Cx} 37^{-/-}$aortas (Fig. 2b). mRNA expression does not necessarily predict protein expression due to for example posttranslational modifications, alternative splicing, etc. [14]. Expression of Cx37 and Cx40 protein content was therefore also assessed by western blotting performed on mouse aortic endothelial cells collected from WT, $\mathrm{Cx} 37^{-1-}$ or $\mathrm{C} \times 40^{-1-}$ mice (see Fig. 2c for a representative example from 6 to 8 mice). These experiments confirmed that $\mathrm{Cx} 37$ was reduced in $\mathrm{Cx}_{4} 0^{-1-}$ aortic endothelial cells as compared to WT endothelium (Fig. 2d, e). Thus, in line with earlier findings in mouse arterioles [15] and the mouse aorta $[9,16], \mathrm{Cx} 37$ was reduced in $\mathrm{Cx}_{40}^{-/-}$endothelial cells. Moreover, in line with yet another study [17], Cx40 expression was not affected by absence of Cx37. Subsequently, the expression of Cx37 and Cx40 was studied in $\mathrm{C} \times 40^{+/-}$ mouse aortic endothelial cells. As expected, these studies 
showed that the expression of $\mathrm{Cx} 40$ tended to be reduced by about $50 \%$ in $\mathrm{Cx} 40^{+/-}$endothelial cells at the level of mRNA (Fig. 2b; $p=0.09$ ) and was significantly reduced at the level of protein (Fig. $2 \mathrm{c}$ and $\mathrm{e} ; p<0.01$ ). However, the expression of $\mathrm{Cx} 37$ in $\mathrm{Cx} 40^{+/-}$aortic endothelial cells was not significantly different from its expression in WT aortic endothelium (Fig. 2a, c and d). In conclusion, expression of $\mathrm{Cx} 40$ seems to be necessary for proper expression of $\mathrm{Cx} 37$ in mouse aortic endothelium. However, even low levels of $\mathrm{Cx} 40$ expression, like in $\mathrm{C} \times 40^{+/-}$mice, are sufficient to maintain $\mathrm{Cx} 37$ expression at a level comparable to WT aortic endothelium. As recently demonstrated [13], maintenance of endothelial Cx37 may depend on the presence of $\mathrm{Cx} 40$ rather than to its channel function. Altogether, by using $\mathrm{Cx} 40^{+/-}$aortas the effect of a specific reduction in $\mathrm{Cx} 40$ expression on vasomotor function can be studied independent from a reduction in Cx37.
Cx37 and Cx40 are in close proximity to eNOS in mouse aortic endothelium

Similar to Cx37 and Cx40, eNOS can also be found at cell-cell interfaces in aortic endothelium. Moreover, Cx37 and Cx40 have been suggested to interact with eNOS in cellular junctions of endothelial cells $[8,9]$. Here, the possible interactions between $\mathrm{Cx} 37, \mathrm{Cx} 40$ and eNOS were studied using in situ proximity ligation assays. Cx37 and eNOS (Fig. 3a-c), and Cx40 and eNOS (Fig. 3d-f) were detected in close proximity at cell-cell interfaces in mouse aortic endothelium. Importantly, no signal was detected in negative controls from which the antibody detecting eNOS was omitted (Fig. 3g-i and j-l). Moreover, interactions between $\mathrm{Cx} 37$ and $\mathrm{Cx} 40$ were detected at intercellular junctions of WT aortic endothelial cells (Additional file 1: Figure S1 A-B) and these were absent between $\mathrm{C} \times 40^{-/-}$endothelial cells (Additional file 1: Figure S1 C-D), further illustrating the specificity
A

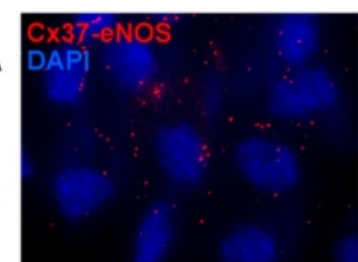

D

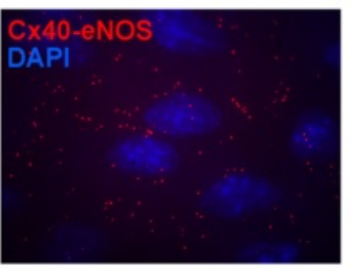

E

$\mathrm{G}$

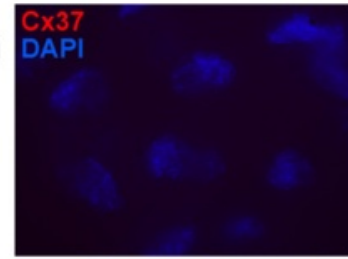

J

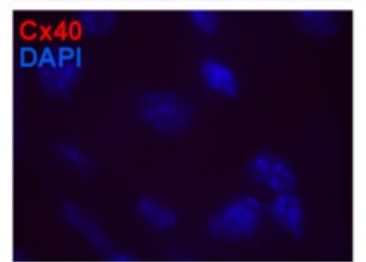

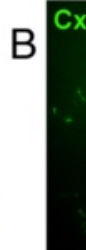

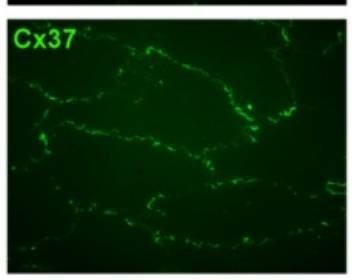

B
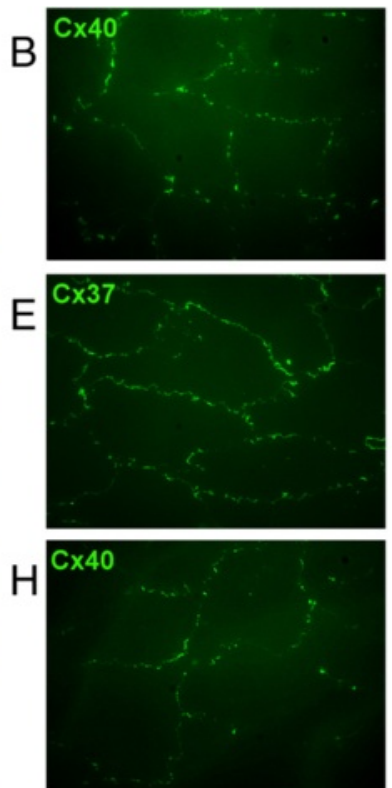

$\mathrm{K}$

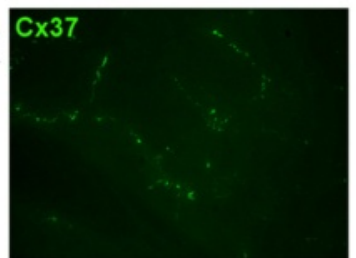

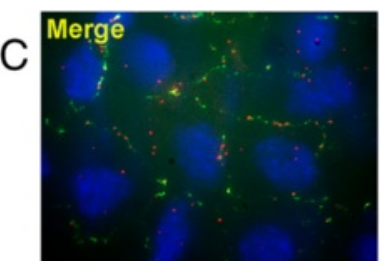

F
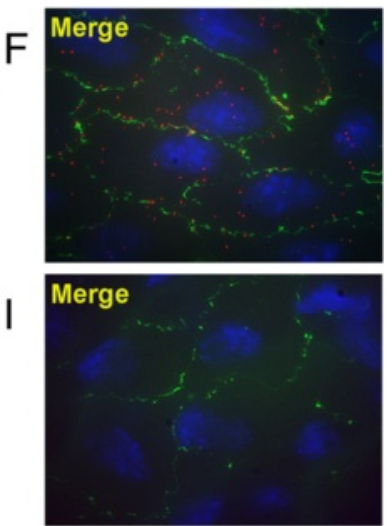

Merge

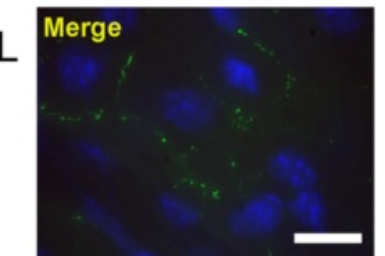

Fig. 3 Interactions between connexins and eNOS are present at cell-cell interfaces between mouse aortic endothelial cells. Representative images of proximity ligation assays performed with antibodies targeting Cx37 and eNOS or Cx40 and eNOS. a Close proximity of Cx37 and eNOS in WT mouse aortic endothelium. $\mathbf{b}$ Cx40 staining to highlight the intercellular gap junctions. c Merge of (a) and (b) highlighting close proximity of eNOS and Cx37 at the gap junctions between endothelial cells. $\mathbf{d}$ Close proximity of Cx40 and eNOS in WT endothelium. e Cx37 staining to highlight the intercellular gap junctions. $\mathbf{f}$ Merge of (d) and (e) highlighting close proximity of eNOS and Cx40 at the gap junctions between endothelial cells. $\mathbf{g}$-i Control assays revealed that the intense red staining observed in a-c was no longer observed after omitting the eNOS antibody from the proximity ligation assays $(\mathbf{g}-\mathbf{i})$ even though cell-cell junctions could clearly be visualized $(\mathbf{h}-\mathbf{i})$. $\mathbf{j}-\mathbf{l}$ : Similar control stainings, which revealed similar results, were performed with the Cx40 antibodies, but not the Cx37 antibodies present during the proximity ligation assays. Scalebar equals $20 \mu \mathrm{M}$ 
of the in situ proximity ligation assays. In conclusion, both $\mathrm{Cx} 37$ and $\mathrm{Cx} 40$ are in close proximity with eNOS at intercellular junctions of mouse aortic endothelial cells. Hence, both Cx37 and Cx40 might regulate eNOSmediated aspects of vasomotor function.

\section{Cx37 is involved in basal NO-mediated vasomotor responses}

The data in this study show that $\mathrm{Cx} 37$ and $\mathrm{Cx} 40$ are i) expressed at cell-cell interfaces and ii) in a close proximity to eNOS. Thus, they suggest that endothelial Cx37 expression may be dependent on proper $\mathrm{Cx} 40$ expression. Moreover, these findings imply that Cx37 and/or Cx40 could regulate eNOS-dependent $\mathrm{NO}$ production in vivo and warrant studies towards a possible role of interactions between $\mathrm{Cx} 37$ or $\mathrm{Cx} 40$ and eNOS in basal NO release at the organ level. Consequently, we assessed phenylephrine (PHE)-induced contractions in aortas isolated from WT, $\mathrm{Cx} 37^{-1-}, \mathrm{Cx} 40^{-1-}$ and $\mathrm{Cx} 40^{+/-}$mice using wire-myography. Initially, PHE-induced in absence of pharmacological inhibitors were studied. These experiments showed that the amplitude of the PHE-induced contractions was significantly enhanced in $\mathrm{Cx} 37^{-/-}$and $\mathrm{C} \times 40^{-/-}$aortas as compared to WT aortas (Fig. 4, Table 1). The amplitude of these contractions, which result from activation of adrenergic receptors expressed by vascular smooth muscle cells, depends on i) the balance between the relative amount of vasoconstrictors and vasodilators present in the organ bath and ii) the amount of vascular smooth muscle cells in the vessel wall. Indeed, as expected, given the hypertensioninduced hypertrophy of the vascular smooth muscle in the $\mathrm{Cx} 40^{-/-}$aortas [9], the PHE-induced contractions were significantly enhanced in the $\mathrm{C} \times 40^{-/-}$aortas as compared to the $\mathrm{Cx} 37^{-/-}$and WT aortas (Fig. 4, Table 1). Interestingly, in case of basal NO release, one would expect that presence of the NOS inhibitor $N^{\omega}$-nitro-l-arginine methyl ester (L-NAME) would increase the contractile amplitude. Indeed, the amplitude of PHE-induced contractions was significantly increased in aortas isolated from WT mice in presence of L-NAME (Fig. 4a, Table 1). This was not the case in aortas isolated from $\mathrm{Cx} 37^{-1-}$ (Fig. 4b, Table 1) or $\mathrm{Cx} 40^{-/-}$mice (Fig. 4c, Table 1). Presence of L-NAME significantly increases PHE-induced contractions in aortas isolated from $\mathrm{Cx} 40^{+/-}$mice that are characterized by a $50 \%$ reduced expression of $\mathrm{Cx} 40$ but normal $\mathrm{Cx} 37$ expression levels (Fig. 4d, Table 1). In conclusion, our observations in $\mathrm{C} \times 37^{-/-}, \mathrm{Cx} 40^{+/-}$and $\mathrm{Cx} 40^{-/-}$aortas show that $\mathrm{Cx} 37$ is implicated in basal NO release. Although it cannot be completely ruled out that the remaining level of $\mathrm{Cx} 40$ expression in $\mathrm{Cx} 40^{+/-}$aortas is sufficient for the generation of basal NO levels comparable to WT aortas, the apparent involvement of $\mathrm{Cx} 40$ is most likely explained by an indirect mechanism (e.g. via the regulation of
Cx37 expression). However, Cx37 seems to be necessary for a direct regulation of eNOS function since basal NO release in $\mathrm{Cx} 37^{-/-}$aortas, which have normal Cx40 expression levels, was reduced. Exactly how Cx37 regulates the basal release of NO is currently unclear. One possibility would be via direct effects on eNOS expression levels. We therefore studied eNOS expression by western blotting of mouse aortic endothelial cell lysates collected from WT, $\mathrm{Cx} 37^{-1-}, \mathrm{C} \times 40^{-1-}$ or $\mathrm{C} \times 40^{+/-}$ mice. In line with previous findings $[9,18]$, eNOS expression was reduced in $\mathrm{Cx} 40^{-/-}$endothelium, but expression levels were similar in $\mathrm{Cx} 37^{-/-}, \mathrm{Cx} 40^{+/-}$and WT mice (Fig. 5e). NO production in the endothelium results from a complex series of interactions between eNOS, $\mathrm{Ca}^{2+}$, calmodulin, caveolin and tetrahydropiopterin [19]. Theoretically, Cx37 could alter e.g. binding of any of these factors to eNOS thereby changing its activity and increasing $\mathrm{NO}$ release. Indeed, the Cterminal tail of Cx37 interacts with eNOS at its reductase domain, a part that also contains a caveolin-1 binding site [8]. Finally, one should keep in mind that "mixed channels" composed of both Cx37 and Cx40 may be involved in regulation of NO release as well.

\section{Involvement of Cx37 or Cx40 in agonist-induced NO release}

Basal and activated NO release do not necessarily depend on similar mechanisms. Findings regarding basal NO release can therefore not always be extrapolated to activated NO release. For that reason, CRCs to acetylcholine (ACh) during PHE-induced pre-contractions in aortas isolated from WT, $\mathrm{Cx} 37^{-1-}, \mathrm{C} \times 40^{-1-}$ or $\mathrm{C} \times 40^{+/-}$ mice were studied. In WT aortas ACh induced relaxations that reached maximal amplitudes at an ACh concentration of $300 \mathrm{nM}$ (Fig. 5a, Table 2). However, in presence of indomethacin (INDO) the CRCs for ACh only reached maximal amplitude at an AChconcentration of $10 \mu \mathrm{M}$ (Fig. 5a, Table 2). Finally, AChinduced relaxations were dramatically reduced in presence of L-NAME (maximal relaxation $-11 \pm 2 \%$ ) and in presence of L-NAME + INDO (Fig. 5a; maximal relaxation $4 \pm 2 \%)$. Thus, in WT aortas ACh-induced relaxations are fully mediated by $\mathrm{NO}$ and can be partially masked by INDO-sensitive contractile factors at high ACh concentrations. Surprisingly, the CRCs for ACh that were generated in $\mathrm{Cx} 37^{-1-}$ aortas were characterized by a lower sensitivity compared to CRCs generated in WT aortas (Table 3) and were unaffected by INDO (Fig. 5b, Table 2). Similar to its effect in WT-aortas, presence of L-NAME drastically reduced the effect of $\mathrm{ACh}$ in $\mathrm{Cx} 37^{-1-}$ aortas (Fig. 5b). CRCs for ACh generated in aortas from $\mathrm{Cx} 40$-deficient animals were also characterized by a lower sensitivity compared to CRCs generated in WT aortas (Table 3). However, INDO 

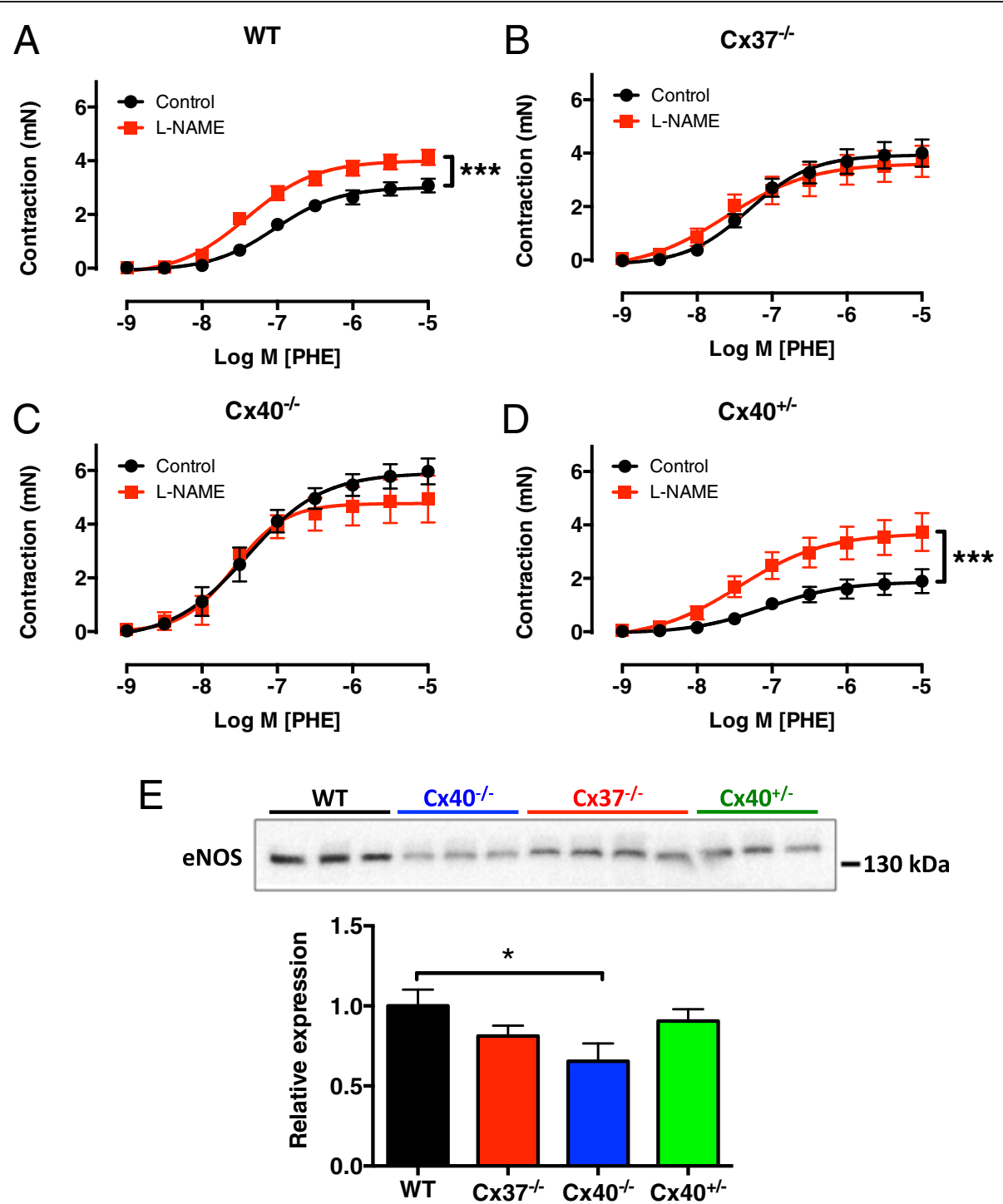

Fig. $4 \mathrm{C} \times 37$ is implicated in basal NO release. Aortic segments obtained from WT, $\mathrm{C} \times 37^{-1-}, \mathrm{C} \times 40^{-/-}$or $\mathrm{C} \times 40^{+/-}$mice were mounted in a wire-myograph and concentration-response curves for PHE were generated in presence or absence of L-NAME (100 $\mu M$ ). a Inhibition of NO synthesis by L-NAME increased the contractile amplitude for PHE in WT aortas indicating presence of basal NO release. This effect of L-NAME was not observed in aortas isolated from $\mathrm{C} \times 37^{-1-}$ (b) or $\mathrm{C} \times 40^{-1-}$ (c) mice. In contrast (d), in aortas obtained from $\mathrm{C} \times 40^{+--}$mice L-NAME did increase the contractile amplitude of PHE. Values are expressed as mean \pm SEM. $N=6-10 .{ }^{*},{ }^{* * *} p<0.01,0.001$ vs control, respectively. e Representative image and quantification of eNOS protein expression in protein samples from scraped mouse aortic endothelial cells as assessed by western blot. Values are expressed as mean \pm SEM. N $=6-8 .{ }^{*} p<0.05$ vs WT

Table 1 Characteristics of PHE-induced contractions in presence or absence of L-NAME

\begin{tabular}{|c|c|c|c|c|c|c|c|c|}
\hline & \multicolumn{4}{|c|}{$E_{\max }(\operatorname{mean}(m N) \pm S E M)$} & \multicolumn{4}{|c|}{$\mathrm{EC}_{50}($ mean $(\mathrm{nM}) 95 \% \mathrm{Cl})$} \\
\hline & $\overline{\mathrm{WT}}$ & $\mathrm{C} \times 37^{-/-}$ & $\mathrm{C} \times 40^{-/-}$ & $\mathrm{C} \times 40^{+/-}$ & $\overline{\mathrm{WT}}$ & $\mathrm{C} \times 37^{-1-}$ & $\mathrm{C} \times 40^{-1-}$ & $\mathrm{C} \times 40^{+/-}$ \\
\hline Control & $3.1 \pm 0.2$ & $4.0 \pm 0.5 \$ \$ \$, \S \S \S$ & $6.0 \pm 0.5 \$ \$ \$$ & $1.9 \pm 0.4 \S \S \S$ & $9195 \%$ Cl $[57,145]$ & $4895 \%$ Cl $[25,94]$ & $3995 \%$ Cl $[20,78]$ & 8495 \% Cl [24, 291] \\
\hline L-NAME & $4.1 \pm 0.3^{* * *}$ & $3.7 \pm 0.6$ & $4.9 \pm 0.9$ & $3.7 \pm 0.7^{* * *}$ & $3995 \%$ Cl $[24,62]$ & $2495 \%$ Cl $[5,115]$ & 2695 \% Cl [12, 57] & $3795 \%$ Cl $[8,160]$ \\
\hline
\end{tabular}

$N=6-10 . * * *$, $\$ \$, \S \S \S p<0.001$ vs the respective control, WT or $\mathrm{C} \times 40^{-/-}$ 

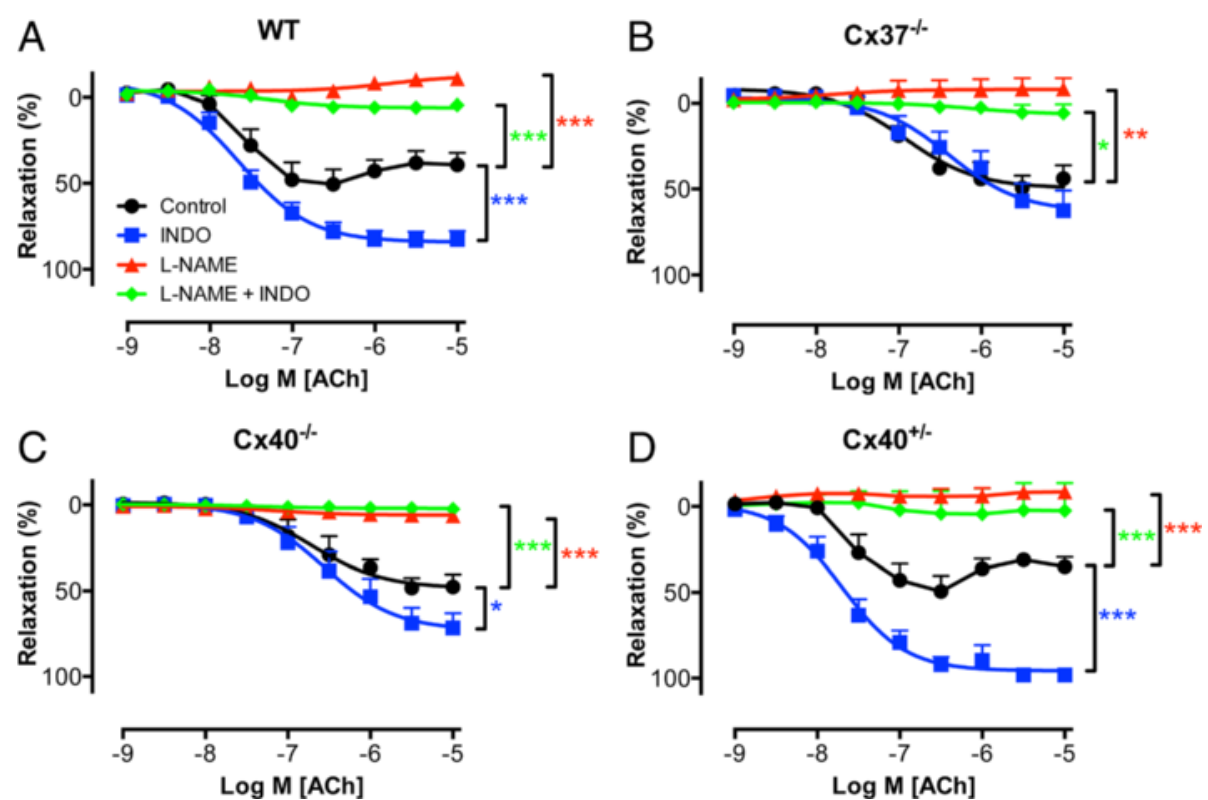

Fig. 5 CX37 may be implicated in the release of COX products but not in activated NO release. Concentration-response curves for the endothelium-specific agonist ACh were generated in PHE pre-contracted aortas obtained from WT, $\mathrm{C} \times 37^{-1-}, \mathrm{C} \times 40^{-1-}$ or $\mathrm{C} \times 40^{+/-}$mice. a WT aortas displayed relaxations in response to ACh (black) which were i) increased in presence of INDO (blue) and ii) inhibited in presence of L-NAME (red) or in presence of L-NAME and INDO (green). $\mathbf{b} \mathrm{C} \times 37^{-/-}$aortas displayed relaxation responses to ACh (black) which were i) unaffected by presence of INDO (blue), ii) inhibited in presence of L-NAME (red) or in presence of L-NAME and INDO (green). c Aortas obtained from Cx40 ${ }^{-1-}$ mice (c) displayed relaxations in response to ACh (black) which were i) slightly, but significantly, increased in presence of INDO (blue) and ii) inhibited in presence of L-NAME (red) or in presence of L-NAME and INDO (green). $\mathbf{d}$ Aortas obtained from $\mathrm{C} \times 40^{+/-}$mice displayed relaxations in response to ACh (black) which were i) increased in presence of INDO (blue) and ii) inhibited in presence of L-NAME (red) or in presence of L-NAME and INDO (green). Values are expressed as mean \pm SEM. $N=6-10 .{ }^{*},{ }^{* *}$ or ${ }^{* * *} p<0.05,0.01$ or 0.001 vs. control, respectively

displayed a slight but significant effect on the maximal effect of ACh in $\mathrm{Cx}_{40} 0^{-/-}$aortas (Fig. 5c, Table 2). In line with expectations, L-NAME also reduced the effects of ACh in the $\mathrm{Cx}_{40} 0^{-1-}$ aortas (Fig. 5c, Table 2). Finally, CRCs for ACh generated in $\mathrm{Cx} 40^{+/-}$aortas were similar in all aspects to those generated in WT aortas (Fig. 5d, Table 2 and Table 3). In summary, Cx37 and Cx40 do probably not affect the activated NO release. Cx37, however, seems implicated in the release of constricting COX products in response to higher ACh concentrations since a significant effect on INDO was not observed in $\mathrm{Cx} 37^{-/-}$aortas (which express Cx40 at WT levels) but was observed in $\mathrm{Cx} 40^{-/-}$ aortas (which express low levels of $\mathrm{Cx} 37$ ) and in $\mathrm{Cx} 40^{+/-}$

Table $2 \mathrm{E}_{\max }$ of ACh-induced relaxations during PHE-induced contractions in presence or absence of INDO, L-NAME or a combination of the former

\begin{tabular}{lllll}
\hline & \multicolumn{4}{l}{$\mathrm{E}_{\max }$ (mean (\% relaxation) \pm SEM) } \\
\cline { 2 - 5 } & WT & $\mathrm{C} \times 37^{-/-}$ & $\mathrm{C} \times 40^{-/-}$ & $\mathrm{C} \times 40^{+/-}$ \\
\hline Control & $51 \pm 9$ & $50 \pm 7$ & $47 \pm 7$ & $50 \pm 9$ \\
INDO & $83 \pm 5^{* * *}$ & $63 \pm 12$ & $71 \pm 8^{*}$ & $98 \pm 3^{* *}$ \\
L-NAME & $-11 \pm 2^{* * *}$ & $-8 \pm 6^{* * *}$ & $6 \pm 4^{* * *}$ & $-8 \pm 4^{* * *}$ \\
L-NAME + INDO & $4 \pm 2^{* * *}$ & $6 \pm 5^{* * *}$ & $2 \pm 3^{* * *}$ & $3 \pm 16^{* *}$ \\
\hline
\end{tabular}

$N=6-10 .{ }^{*}{ }^{* *}$ or ${ }^{* * *} p<0.05,0.01$ or 0.001 vs control aortas (which express Cx37 at WT levels). Furthermore, changes in the expression of Cx37 are most likely also responsible for the changes in the sensitivity for ACh that were observed since these changes were fully normalized in $\mathrm{Cx} 4 \mathrm{O}^{+/-}$aortas. However, half of $\mathrm{Cx} 40$ expression may also be sufficient for normal endothelial function. Surprisingly, ACh-induced NO-mediated dilatation was not affected by Cx40 (or by Cx37; this study) in contrast to earlier work from our group [9]. The reasons for this discrepancy are not entirely clear but are most likely due to methodological differences between the studies (e.g. the use of different agonists to induce precontractions, cumulative $v s$ noncumulative CRCs, different timing of agonist application).

\section{Involvement of Cx37 or Cx40 in release of contractile factors}

Various studies have described endothelium-dependent contractile effects of high ACh concentrations [20-23]. Indeed, in the current study the lower ACh concentrations induced relaxations that no longer increased at higher ACh concentrations in WT aortas. It has been shown that COX1-derived products mediate contractile effects of ACh in the mouse aorta [20]. We therefore assessed the effect of blocking cyclooxygenases using INDO (a dual COX1/COX2 inhibitor) on ACh-induced 
Table $3 \mathrm{EC}_{50}$ (mean $(\mathrm{nM}) 95 \% \mathrm{Cl}$ ) for ACh during PHE-induced contraction in presence of INDO

\begin{tabular}{llll}
\hline WT & $C \times 37^{-/-}$ & $C \times 40^{-/-}$ & $C \times 40^{+/-}$ \\
\hline $2395 \% \mathrm{Cl}[15,35]$ & $40995 \% \mathrm{Cl}[153,1088]^{*}$ & $27495 \% \mathrm{Cl}[125,604]^{*}$ & $1995 \% \mathrm{Cl}[11,32]$ \\
\hline${ }^{*} p<0.05$ vs WT & &
\end{tabular}

relaxations. Indeed, presence of INDO significantly increased the maximal effect of ACh in WT, $\mathrm{Cx} 40^{-/-}$and $\mathrm{Cx} 40^{+/-}$but not $\mathrm{Cx} 37^{-/-}$aortas. Interestingly, it has been suggested that gap junctional communication between endothelial cells or, alternatively, between endothelium and smooth muscle cells could be responsible for the contractile effects of $\mathrm{ACh}$ in the aorta of spontaneouslyhypertensive rats [21]. However, this latter study relied on rather non-specific chemical inhibitors such as carbenoxolone and only addressed the role of connexins in the context of hypertension, a disease that is well-known to alter the distribution of connexins in the vascular wall [24]. As effects of INDO on the Emax of ACh-induced relaxations was observed in $\mathrm{WT}, \mathrm{Cx} 40^{+/-}$and $\mathrm{Cx} 40^{-1-}$ aortas but not in $\mathrm{Cx} 37^{-1-}$ aortas, we conclude that $\mathrm{Cx} 37$ is necessary to allow the effect of INDO.

\section{Connexin deficiency does not alter the sensitivity for NO}

The altered sensitivity for ACh that was observed in $\mathrm{Cx} 37^{-/-}$and $\mathrm{Cx} 40^{-/-}$aortas may be due to i) a changed sensitivity of the aortic endothelial cells for $\mathrm{ACh}$, ii) an altered eNOS expression level (in the $\mathrm{Cx} 40^{-/-}$but not the $\mathrm{Cx} 37^{-/-}$aortas) or iii) a changed sensitivity of the vascular smooth muscle cells for NO. The latter possibility was tested by generating CRCs to an NO donor (sodium-nitroprusside, (SNP)) during PHE-induced precontractions (in presence of L-NAME and INDO to avoid effects of endogenous $\mathrm{NO}$ or COX-products, respectively) in WT, $\mathrm{Cx} 37^{-/-}, \mathrm{C} \times 40^{-/-}$or $\mathrm{C} \times 40^{+/-}$aortas. These experiments showed that aortas isolated from WT, $\mathrm{Cx} 37^{-/-}, \mathrm{C} \times 40^{-/-}$or $\mathrm{C} \times 40^{+/-}$mice were equally sensitive to NO (Table 4). In summary, the changed sensitivity for ACh, which was observed in $\mathrm{Cx} 37^{-/-}$and $\mathrm{C} \times 40^{-/-}$aortas, was not due to alterations at the smooth muscle level (e.g. a decreased sensitivity of the 'NO receptor' soluble guanylyl cyclase (sGC)) but likely reflected endothelial changes. Indeed, involvement of Cx40 (and most likely $\mathrm{Cx} 37$ ) in the endothelial $\mathrm{Ca}^{2+}$ agonist responses, which govern NO release, are agonist specific [25]. I.e. ATP-induced increases in intracellular $\mathrm{Ca}^{2+}$ in mouse endothelium are homogeneous and do not require synchronization by connexins [25]. In contrast, $\mathrm{Ca}^{2+}$ elevations mediated by $\mathrm{ACh}$ are heterogenous

Table $4 \mathrm{EC}_{50}$ (mean (nM) $95 \% \mathrm{Cl}$ ) for SNP during PHE-induced contraction in presence of L-NAME and INDO

\begin{tabular}{llll}
\hline WT & $\mathrm{C} \times 37^{-/-}$ & $\mathrm{C} \times 40^{-/-}$ & $\mathrm{C} \times 40^{+/-}$ \\
\hline $795 \% \mathrm{Cl}[4,14]$ & $595 \% \mathrm{Cl}[2,10]$ & $395 \% \mathrm{Cl}[1,30]$ & $495 \% \mathrm{Cl}[1,20]$ \\
\hline
\end{tabular}

and require synchronization by connexins to reach other cells [25]. This observation sparks thoughts such as 'thus, the diminished response to ACh makes sense since ACh-induced responses require synchronization via connexins'. However, although this is theory true for the total amount of NO released by aortic endothelial cells exposed to a certain ACh concentration (an effect that was not actually observed in this study), this theory does not hold regarding sensitivity changes. Such changes most likely involve conformational changes of the M3receptor or enzymes, like eNOS, functioning downstream of M3-receptor activation but upstream of the activation of sGC. eNOS seems a likely candidate since Cx37 and Cx40 have not been shown to interact with M3 receptors (nor do they co-localize with M3 receptors in mouse aortic endothelial cells (see Fig. 4 in [25])) but do interact with this NO-generating enzyme. Tantalizingly, if the basal intracellular $\mathrm{Ca}^{2+}$ concentration is unaffected by genotype then a decreased eNOS sensitivity for $\mathrm{Ca}^{2+}$ could explain both the effect observed regarding basal NO release (decreased NO released at fixed $\mathrm{Ca}^{2+}$ concentration) and the apparent effect on sensitivity of ACh (increasing concentrations of ACh result in increasing concentrations of intracellular $\mathrm{Ca}^{2+}$ that do not have similar effects due to the altered $\mathrm{Ca}^{2+}$ sensitivity of eNOS). Obviously, this will be the topic of further studies.

\section{Conclusions}

In summary, the current study was conducted to address whether Cx37 or Cx40 is more pronouncedly involved in the regulation of vasomotor function. The data indicate that Cx37 is more markedly involved in basal NO release, release of COX-products and the regulation of the sensitivity for ACh as compared to Cx40. These findings are exciting since they, for the first time, allow for a discrimination of the functional role of Cx37 and Cx40 in mouse aortic endothelium. However, various questions remain e.g. 'How does $\mathrm{Cx} 37$ modulate basal NO release without modulating eNOS expression levels?, and importantly, 'Why is the sensitivity for ACh altered in $\mathrm{C} \times 37^{-1-}$ aortas'. Clearly, these questions warrant a specific exploration in further studies.

\section{Methods}

The Swiss Federal Veterinary Office approved animal housing and animal experiments. Wild-type (WT, $n=10$ ), $\mathrm{Cx}^{-1-}(n=10), \mathrm{Cx}^{-1} 0^{-/-}(n=8)$ and $\mathrm{Cx}_{40} 0^{+-}(n=8)$ mice were progeny of the original colony on a $\mathrm{C} 57 \mathrm{BL} / 6 \mathrm{~J}$ 
Table 5 Contraction $(\mathrm{mN})$ upon exposure to $\mathrm{K}^{+}(40 \mathrm{mM})$ before exposure to pharmacological inhibitors

\begin{tabular}{llll}
\hline $\mathrm{WT}$ & $\mathrm{C} \times 37^{-/-}$ & $\mathrm{C} \times 40^{-/-}$ & $\mathrm{C} \times 40^{+/-}$ \\
\hline $1.8 \pm 0.2$ & $2.8 \pm 0.3^{*}$ & $3.9 \pm 0.3^{* * *}$ & $1.8 \pm 0.2$ \\
\hline$N=6-10 .{ }^{*}$ or ${ }^{* * *} p<0.05$ or 0.001 vs WT & &
\end{tabular}

background and were housed in a 12-hours day-night cycle [26, 27]. Genotyping was performed as previously described $[17,9]$.

\section{Immunofluorescence and in situ proximity ligation assays}

Immunostainings and in situ proximity ligation assays were performed as previously described $[9,11]$. In brief, for immunostainings, opened thoracic aortas were fixed in ice-cold methanol, permeabilized with $0.2 \%$ TritonX100 and blocked in $2 \%$ bovine serum albumin. Primary antibodies (polyclonal rabbit anti-Cx37 (alpha Diagnostic), anti-Cx40 (Chemicon), anti-Cx43 (alpha Diagnostic) were incubated overnight. An Alexa Fluor 488 fluorochrome-conjugated goat-anti rabbit was used for signal detection. Nucleic acids were stained with DAPI and the cytosol was visualized using Evans Blue. Finally, sections were mounted and analysed using a LSM 510 Meta confocal microscope (Zeiss). For the proximity ligation assays, longitudinally opened aortas were fixed in $100 \%$ ethanol, and aortic strips prepared for the simultaneous incubation with rabbit polyclonal antibody against Cx40 (Chemicon) or Cx37 (Biotrend Chemikalien) and mouse monoclonal antibody against eNOS (BD Bioscience). The incubation solution was then supplemented with oligonucleotide-conjugated secondary antibodies (diluted 1:5) and a ligation solution, consisting of two nucleotides and a ligase, to form a circular DNA when eNOS and $\mathrm{Cx} 40$ or $\mathrm{Cx} 37$ were in close proximity. The circular DNA was then amplified into a long, single-stranded concatemer by rolling circle amplification, using as primer an arm of one of the oligonucleotide-conjugated antibodies. The amplification product was collapsed into a DNA bundle, and detected by hybridizing fluorophore-labeled oligonucleotides (Duolink in situ detection reagent red) to the repeated sequences of the amplification product. Under a fluorescence microscope, the result was detected as red spots, which were localized by staining the EC nuclei with DAPI. For the proximity ligation assays performed with antibodies targeting $\mathrm{Cx} 40$ and $\mathrm{Cx} 37$ in the en face aortic strips, a monoclonal antibody specific to mouse Cx40 was used (Invitrogen).

\section{Quantitative PCR}

RNA isolation, DNAse treatment and reverse transcriptase reactions were performed on whole mouse aortas as previously described [11]. Equal loading was ensured by assessing expression of $18 \mathrm{~S}$ ribosomal RNA and was similar in all samples. The relative amount of mRNA of interest was calculated after normalization to CD31 for the amount of endothelium in the samples.

\section{Western blotting}

The expression of $\mathrm{Cx} 37$ and $\mathrm{Cx} 40$ was determined as previously described [9]. In brief, thoracic aortas were longitudinally opened in $100 \mu \mathrm{l}$ PBS, and were subsequently pinned on a silicone plate with the endothelial-side facing upwards. A scalpel blade was then used to gently scrape off the ECs. ECs in PBS were then centrifuged at $14,000 \mathrm{rpm}$ for $5 \mathrm{~min}$ at $4{ }^{\circ} \mathrm{C}$ and the pellet was homogenized in lysis buffer $(62.5 \mathrm{mM}$ Tris $\mathrm{HCl}$, pH6.8, 5 \% SDS, 10 mM EDTA, pH8). Protein content was measured using a detergent-compatible DC protein assay kit (Bio-Rad Laboratories). Samples $(20 \mu \mathrm{g})$ were equally loaded on a $10 \%$ polyacrylamide gel, separated by electrophoresis and transferred onto PVDF membranes (Immobilon-P; Millipore). Membranes were incubated for $1 \mathrm{~h}$ in PBS or TBS containing $5 \%$ milk and $0.1 \%$ Tween 20 (blocking buffer). The membranes were then incubated overnight at $4{ }^{\circ} \mathrm{C}$ with one of the following primary antibodies: rabbit polyclonal antibodies against Cx40 (Chemicon), Cx37 (Biotrend Chemikalien) or von Willebrand factor (vWF) (Dako) and mouse monoclonal antibodies against $\alpha$-tubulin (Sigma-Aldrich) or eNOS (BD Bioscience). The secondary antibodies were horseradish peroxidase-conjugated goat anti-mouse immunoglobulin (Jackson Immuno research); or goat anti-rabbit immunoglobulin (Thermo Scientific), respectively. Bands were developed using enhanced chemiluminescence (Millipore), and visualized using a supercooled CCD camera (Chemidoc XRS). Densitometric analysis was performed using ImageLab Software (3.0.1 Bio-Rad Laboratories). Equal loading was ensured by assessing expression of tubulin and was similar in all the samples. The relative amount of the proteins of interest was calculated after normalization to vWf for the amount of endothelium in the samples.

\section{Solutions and drugs}

Krebs-Ringer bicarbonate-buffered physiological salt buffer (KRB-buffer) contained $118.5 \mathrm{mM} \mathrm{NaCl}, 4.7 \mathrm{mM}$ $\mathrm{KCl}, 2.5 \mathrm{mM} \mathrm{CaCl}_{2}, 1.2 \mathrm{mM} \mathrm{MgSO} 4,1.2 \mathrm{mM} \mathrm{KH_{2 }} \mathrm{PO}_{4}$, $25.0 \mathrm{mM} \mathrm{NaHCO} 3$ and $5.5 \mathrm{mM}$ glucose. High $\mathrm{K}^{+}-\mathrm{KRB}$ solution was prepared by replacing $\mathrm{NaCl}$ with $\mathrm{KCl}$. Buffers containing intermediate $\mathrm{K}^{+}$concentrations were prepared by mixing volumes of $\mathrm{KRB}$ and $\mathrm{K}^{+}-\mathrm{KRB}$. Acetylcholine (ACh), phenylephrine (PHE), $N^{\omega}$-nitro-larginine methyl ester (L-NAME), sodium-nitroprusside (SNP) and indomethacin (INDO) were obtained from Sigma-Aldrich (Germany). All compounds were dissolved in KRB-buffer with the exception of INDO, which was dissolved in ethanol. 


\section{Wire-myography}

Mice were sedated (ketamine (100 mg/kg) + xylazine (100 mg/kg), i.p. injection). Subsequently, the left ventricle was punctured with a $23 \mathrm{G}$ needle. Then, the animals were perfused during $3 \mathrm{~min}$ under constant flow (flow rate $3.33 \mathrm{~mL} / \mathrm{min}$ ) with a total volume of $10 \mathrm{~mL} \mathrm{KRB-buffer} \mathrm{(pre-heated} \mathrm{at} 37{ }^{\circ} \mathrm{C}$ ). After perfusion, aortic segments were isolated and mounted in a multi-myograph (610 M; DMT, Aarhus, Denmark). After mounting, the segments were stretched to the diameter at which maximal contractile responses to $40 \mathrm{mM} \mathrm{K}^{+}\left(\mathrm{K}_{\max }\right)$ were obtained (Table 5). Next, to test endothelial integrity, arteries were washed precontracted using PHE $(10 \mu \mathrm{M})$ and subsequently exposed to ACh $(10 \mu \mathrm{M})$. Within each genotype, all segments displayed relaxing responses of equal magnitude in response to this muscarinic receptor agonist (data not shown). Thereafter, a concentration-response curve (CRC) for PHE $(1 \mathrm{nM}-10 \mu \mathrm{M})$ was generated. Subsequently, during the stabilized contractile response to $10 \mu \mathrm{M}$ PHE, a CRC for ACh $(1 \mathrm{nM}-10 \mu \mathrm{M})$ was constructed. Twenty minutes after the CRC for ACh the arterial segments were again precontracted using PHE $(10 \mu \mathrm{M})$. Then, in order to test the NO sensitivity of the vascular smooth muscle cells in the preparation, a CRC for SNP $(0.1 \mathrm{nM}-10 \mu \mathrm{M})$ was generated during the stabilized contractile response to PHE. These experiments were conducted in parallel in presence of $\mathrm{L}$ NAME (100 $\mu \mathrm{M}$; to block the synthesis of NO), INDO (10 $\mu \mathrm{M}$; to block the synthesis of prostaglandins) or a combination of the former.

\section{Statistical analyses}

Results are shown as mean \pm SEM. One or two-way ANOVAs followed by Bonferroni's multiple comparisons tests were performed using Graphpad Prism 6.0 to compare mean values between the groups. $P$-values $<0.05$ were considered statistically significant.

\section{Additional file}

Additional file 1: Interactions between Cx37 and Cx40 at cell-cell interfaces between mouse aortic endothelial cells.

\footnotetext{
Abbreviations

ACh: Acetylcholine; ANOVA: Analysis of variance; ATP: Adenosine triphosphate; CAMP: cyclic adenosine monophosphate; CRC: Concentration-response curve; Cx37: Connexin37; Cx40: Connexin40; DNAse: Deoxyribonuclease; eNOS: endothelial nitric oxide synthase; INDO: Indomethacin; KRB-buffer: Krebs-Ringers bicarbonate-buffered physiological salt buffer; L-NAME: $N^{\omega}$ -nitro-l-arginine methyl ester; NO: Nitric oxide; $\mathrm{PGI}_{2}$ : Prostacyclin; PHE: Phenylephrine; RNA: Ribonucleic acid; sGC: soluble guanylyl cyclase; SNP: Sodium-nitroprusside; vWf: von Willebrand factor; WT: Wild-type.
}

\section{Competing interests}

The authors declare that they have no competing interests.

\section{Authors' contributions}

MJM, FA and LLG performed the research. MJM, BRK and J-AH designed the research study. MJM, FA and LLG analysed the data. MJM, BRK and J-AH wrote the paper. All authors read and approved the final manuscript.

\section{Acknowledgements}

This work was supported by a grant from the Swiss National Science Foundation (grant number 310030_143343/1 to BRK, 31003A-155897 to JAH), the Octav and the Marcella Botnar Foundation.

\section{Author details}

${ }^{1}$ Department of Pathology and Immunology, University of Geneva, 6th floor, 1 Rue Michel-Servet, 1211 Geneva, Switzerland. ²Department of Medical Specialties - Cardiology, University of Geneva,

Geneva, Switzerland. 'epartment of Medicine, University Hospital, CHUV, Lausanne, Switzerland.

Received: 27 April 2015 Accepted: 3 July 2015

Published online: 22 July 2015

\section{References}

1. Pfenniger A, Wohlwend A, Kwak BR. Mutations in connexin genes and disease. Eur J Clin Investig. 2011;41(1):103-16. doi:10.1111/j. 1365-2362.2010.02378.x.

2. Dobrowolski R, Willecke K. Connexin-caused genetic diseases and corresponding mouse models. Antioxid Redox Signal. 2009;11(2):283-95. doi:10.1089/ars.2008.2128.

3. Bosco D, Haefliger JA, Meda P. Connexins: key mediators of endocrine function. Physiol Rev. 2011;91(4):1393-445. doi:10.1152/physrev.00027.2010.

4. Saez JC, Leybaert L. Hunting for connexin hemichannels. FEBS letters. 2014 doi:10.1016/j.febslet.2014.03.004

5. Agullo-Pascual E, Cerrone M, Delmar M. Arrhythmogenic cardiomyopathy and Brugada syndrome: diseases of the connexome. FEBS Lett. 2014;588(8):1322-30. doi:10.1016/j.febslet.2014.02.008.

6. Laird DW. The gap junction proteome and its relationship to disease. Trends Cell Biol. 2010;20(2):92-101. doi:10.1016/j.tcb.2009.11.001.

7. Herve JC. The communicating junctions, composition, structure and characteristics. Biochim Biophys Acta. 2012;1818(8):1803-6. doi:10.1016/j.bbamem.2012.05.008.

8. Pfenniger A, Derouette JP, Verma V, Lin X, Foglia B, Coombs W, et al. Gap junction protein $\mathrm{C} \times 37$ interacts with endothelial nitric oxide synthase in endothelial cells. Arterioscler Thromb Vasc Biol. 2010;30(4):827-34. doi:10.1161/ATVBAHA.109.200816.

9. Alonso F, Boittin FX, Beny $J$, Haefliger JA. Loss of connexin40 is associated with decreased endothelium-dependent relaxations and eNOS levels in the mouse aorta. Am J Physiol Heart Circ Physiol. 2010;299(5):H1365-73. doi:10.1152/ajpheart.00029.2010.

10. Gabriels JE, Paul DL. Connexin43 is highly localized to sites of disturbed flow in rat aortic endothelium but connexin37 and connexin40 are more uniformly distributed. Circ Res. 1998;83(6):636-43.

11. Pfenniger A, Wong C, Sutter E, Cuhlmann S, Dunoyer-Geindre S, Mach F, et al. Shear stress modulates the expression of the atheroprotective protein Cx37 in endothelial cells. J Mol Cell Cardiol. 2012;53(2):299-309. doi:10.1016/j.yjmcc.2012.05.011.

12. Vorderwulbecke BJ, Maroski J, Fiedorowicz K, Da Silva-Azevedo L, Marki A, Pries $A R$, et al. Regulation of endothelial connexin40 expression by shear stress. Am J Physiol Heart Circ Physiol. 2012;302(1):H143-52. doi:10.1152/ajpheart.00634.2011.

13. Jobs A, Schmidt K, Schmidt VJ, Lubkemeier I, van Veen TA, Kurtz A, et al. Defective Cx40 maintains Cx37 expression but intact Cx40 is crucial for conducted dilations irrespective of hypertension. Hypertension. 2012;60(6):1422-9. doi:10.1161/HYPERTENSIONAHA.112.201194.

14. Vogel C, Marcotte EM. Insights into the regulation of protein abundance from proteomic and transcriptomic analyses. Nat Rev Genet. 2012;13(4): 227-32. doi:10.1038/nrg3185

15. de Wit C. Different pathways with distinct properties conduct dilations in the microcirculation in vivo. Cardiovasc Res. 2010:85(3):604-13. doi:10.1093/cvr/cvp340. 
16. Chadjichristos $C E$, Scheckenbach $K E$, van Veen TA, Richani Sarieddine MZ, de Wit C, Yang Z, et al. Endothelial-specific deletion of connexin40 promotes atherosclerosis by increasing CD73-dependent leukocyte adhesion. Circulation. 2010;121(1):123-31. doi:10.1161/CIRCULATIONAHA.109.867176.

17. Wong CW, Christen T, Roth I, Chadjichristos CE, Derouette JP, Foglia BF,

et al. Connexin37 protects against atherosclerosis by regulating monocyte adhesion. Nat Med. 2006;12(8):950-4. doi:10.1038/nm1441.

18. Le Gal L, Alonso F, Mazzolai L, Meda P, Haefliger JA. Interplay between connexin40 and nitric oxide signaling during hypertension. Hypertension. 2015;65(4):910-5. doi:10.1161/HYPERTENSIONAHA.114.04775.

19. Fleming I, Busse R. Signal transduction of eNOS activation. Cardiovasc Res. 1999;43(3):532-41.

20. Tang EH, Ku DD, Tipoe GL, Feletou M, Man RY, Vanhoutte PM Endothelium-dependent contractions occur in the aorta of wild-type and COX2-/- knockout but not COX1-/- knockout mice. J Cardiovasc Pharmacol. 2005;46(6):761-5.

21. Tang EH, Vanhoutte PM. Gap junction inhibitors reduce endothelium-dependent contractions in the aorta of spontaneously hypertensive rats. J Pharmacol Exp Ther. 2008:327(1):148-53. doi:10.1124/jpet.108.140046.

22. De Mey JG, Vanhoutte PM. Heterogeneous behavior of the canine arterial and venous wall. Importance of the endothelium. Circ Res. 1982;51(4):439-47.

23. Baretella O, Xu A, Vanhoutte PM. Acidosis prevents and alkalosis augments endothelium-dependent contractions in mouse arteries. Pflugers Arch - Eur J Physiol. 2014;466(2):295-305. doi:10.1007/s00424-013-1323-z.

24. Figueroa XF, Isakson BE, Duling BR. Vascular gap junctions in hypertension. Hypertension. 2006;48(5):804-11. doi:10.1161/01.HYP.0000242483.03361.da.

25. Boittin FX, Alonso F, Le Gal L, Allagnat F, Beny JL, Haefliger JA. Connexins and $\mathrm{M} 3$ muscarinic receptors contribute to heterogeneous $\mathrm{Ca}(2+)$ signaling in mouse aortic endothelium. Cell Physiol Biochem. 2013;31(1):166-78. doi:10.1159/000343358

26. Simon AM, Goodenough DA, Li E, Paul DL. Female infertility in mice lacking connexin 37. Nature. 1997:385(6616):525-9. doi:10.1038/385525a0.

27. Kirchhoff S, Nelles E, Hagendorff A, Kruger O, Traub O, Willecke K. Reduced cardiac conduction velocity and predisposition to arrhythmias in connexin40-deficient mice. Curr Biol. 1998;8(5):299-302.

\section{Submit your next manuscript to BioMed Central and take full advantage of:}

- Convenient online submission

- Thorough peer review

- No space constraints or color figure charges

- Immediate publication on acceptance

- Inclusion in PubMed, CAS, Scopus and Google Scholar

- Research which is freely available for redistribution

Submit your manuscript at www.biomedcentral.com/submit 